\title{
Sleep and Menopause: Review
} \author{
Marina $^{6}$ \\ 1,4,5,6 Department of Gynecological Endocrinology and Climacteric Section, Hospital Italiano de Buenos Aires \\ ${ }^{2}$ Department of Gynecology, Hospital Italiano de Buenos Aires \\ ${ }^{3}$ Chief of Gynecological Endocrinology and Climacteric Section, Hospital Italiano de Buenos Aires
}

Starvaggi Agustina ${ }^{1 *}$, Pilnik Susana ${ }^{2}$, Belardo Maria Alejandra ${ }^{3}$, Cavanna Malena ${ }^{4}$, De Nardo Barbara ${ }^{5}$ and Gelin

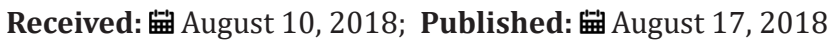

*Corresponding author: Starvaggi Agustina, Department of Gynecology, Italiano de Buenos Aires Hospital, Argentina

Abbreviations: MHT: Hormone Replacement Therapy; ICSD: International Classification of Sleep Disorders; RF: Risk Factors; AIS: Athens Insomnia Scale; PSQI: Pittsburgh Sleep Quality Index; MRS: Menopause Rating Scale; SL: Sleep Latency; SWS: Slow Wave Sleep; PSG: Polysomnography

\section{Introduction}

\section{Objectives}

a. To recognize sleep characteristics and their classification

b. To assess the prevalence and type of sleep disturbances most frequent in this stage of life

c. To evaluate the relationship between sleep disturbances and vasomotor symptoms and the role of hormone replacement therapy of menopause (MHT) plays in preventing them.

\section{Sleep Characteristics and Classification of Sleep Disturbances}

Sleep consists of REM and non-REM (rapid eye movement) periods that happen cyclically. Each one lasts between 90 to 120 minutes, and in one night there are approximately 4 to 6 cycles. During the first half of the night, non-REM cycles predominate, which are related to physical and mental restitution. On the contrary, in the second half of the night, REM cycles predominate, which participate in mental and emotional functions, including memory [1].

According to ICSD-3 (International Classification of Sleep Disorders), sleep disturbances are classified as it shows on Figure 1 [2]. Insomnia is, by far, the most frequent condition, and this disorder includes any disorder in the conciliation or maintenance of sleep that produces diurnal disorders. Within respiratory disorders, there is the sleep apnea, which is defined as repeated episodes of collapse of the upper airway during sleep. This results in sleep quality deterioration due to intermittent hypoxemia, decreased REM sleep and slow wave sleep, which is important for memory.
Affected patients complain of daytime sleepiness, insomnia and numerous of other symptoms (snoring, gasping, choking, memory impairment) leading to an impaired quality of life. The risk factors (RF) for its development are age, male sex and the presence of android obesity. Postmenopausal women present 2.6 to 3.5 times more risk than premenopausal women to present it. Physiological factors explaining this increased prevalence include weight gain, fat distribution changes due to increased testosterone production and a decreased level of circulating female gonadal hormones. Without treatment, it constitutes an independent RF for the development of hypertension, cardiovascular disease, arrhythmia, stroke and therefore an increase in mortality [3]. And finally, less frequent disorders such as narcolepsy (within the group of hypersomnia), parasomnias (REM / non-REM) or restless legs syndrome (within movement disorders).

- Insomnia

- Respiratory disorders related to sleep

- Disorders related to hypersomnia of central origin

- Circadian rhythm disorders

- Parasomnias

- Movement disorders related to sleep

- Other sleep disorders

Figure 1: Classification of sleep disorders according to ICSD-3 (International Classification of Sleep Disorders) [2].

Prevalence of Sleep Disorders in the Peri and Post menopause

Regarding the prevalence of sleep disorders during the transition to menopause and post menopause, the large studies 
showed an increase in it, specifically in this period of life. The WHI study showed a prevalence of $54 \%$ in the group of women aged 45-59 years [4], while the SWAN study, which included women between 40-55 years, 38\% [5]. Another large Japanese study, which included more than 1400 women, demonstrated a prevalence like the WHI: 50.8\% insomnia in peri and postmenopausal women [6]. Regarding the scientific evidence in Latin American women, a study that included 6079 women between 40 and 59 years old from 11 countries of Latin America was carried out [7], whose objective was to determine the prevalence of sleep disturbances in middle-aged women using validated scales. The questionnaires included were the Athens Insomnia Scale (AIS), a self-filling questionnaire to assess sleep disorders, the Pittsburgh Sleep Quality Index (PSQI), another self-filling questionnaire that evaluates the quality of sleep in the last 4 weeks, the Menopause Rating Scale (MRS), a questionnaire that identifies the frequency and intensity of climacteric symptoms divided into 3 domains, Goldberg Anxiety and Depression Scale and Brief Scale of Abnormal Drinking, to rule out anxiety or depression and inadequate alcohol consumption, respectively. In that study, they found that $56.6 \%$ of women had some type of sleep disturbance (insomnia, poor sleep quality or both). The prevalence of Insomnia increased $39.7 \%$ in the group of younger women (40-44 years) to $45.2 \%$ in the group of postmenopausal women (55-59 years) with a significant difference between both groups $(\mathrm{p}=0.009)$. Regarding sleep quality, the prevalence of poor sleep quality increased from $40.3 \%$ in the first group to $49.3 \%$ in the second, with an also significant difference $(\mathrm{p}=0.0001)$.

Regarding the scales, the AIS presented an increase in the total postmenopausal score from 4.90 to $5.81(\mathrm{p}=0.0001)$ and of all the individual items, with nighttime awakening being the highest score and difficulty in induction of sleep the greatest increase $(32.8 \%)$ in postmenopausal women. Regarding the PSQI, an increase in the total postmenopausal score from 4.28 to 5.30 was also observed ( $\mathrm{p}=0.0001$ ), with the drowsiness item having a peak in peri and early post menopause. In postmenopausal women, the item "medication requirement" (increased by 55.9\%) and "sleep latency" (increased by $25.3 \%$ ) increased significantly.

\section{Mechanisms associated with Climacteric Sleep Changes}

During menopause, there are changes in 4 different neurotransmitter systems, such as the GABAergic, serotonergic (5HT), noradrenergic (NA) and dopaminergic (DA) systems. Both NA and DA are involved in sleep regulation. Estrogen-dependent changes in serotonin during the transition to menopause may be responsible for changes in sleep, mood and memory. In fact, experimental models confirm that the depletion of 5HT levels in the brain causes insomnia [8]. The impact of hormonal depletion in sleep is demonstrated in clinical studies. In a study conducted in Pennsylvania [9], 1324 healthy volunteers without sleep complaints who spent a night in the sleep laboratory were included. Among other parameters, the total sleep time (as a percentage of bed time,
$\%$ ST) was evaluated. They observed that in men there was a linear reduction of \% ST with age; On the contrary, in women there was a non-significant decrease of \% ST with age in women under 50 followed by a significant decrease in those over 50 years.

\section{What is the Relationship between sleep disturbances and vasomotor symptoms?}

For a long time was discussed whether if hot flashes disturb sleep, or if there is a sleep disturbance during post menopause, with more frequent awakenings, after which the hot flashes occur. The order of this sequence is related to the type of sleep cycle. REM sleep suppresses thermoregulatory responses; therefore, during the first part of the night, the sequence is hot flash and the awakening afterwards (due to the predominance of non-REM cycles). On the other hand, during the second part, the sequence is in the reverse order (awakening and then suffocation). On the Latin American study previously mentioned, a directly relationship was observed between intensity of hot flushes (item 1 of MRS), AIS and PSQI score. Regarding the rest of climacteric symptoms, a directly relationship was found between total MRS score, insomnia prevalence and poor sleep quality [7].

\section{Sleep and Menopause: Our Experience}

\section{MHT: What role does it play?}

Although the climacteric symptoms are not the unique cause of sleep disorders in the peri and post menopause period, the MHT in symptomatic women could contribute to improve the sleep quality. In the Pennsylvania study previously mentioned [9], differences were observed in some sleep parameters such as sleep latency (SL) and slow wave sleep (SWS), which constitutes a non-REM sleep phase and is related to memory. The lower SL and the greater SWS, the better is the sleep quality. In this study we found a higher SL and decreased SWS in the group of postmenopausal women without MHT compared to postmenopausal women with MHT and men. SL in postmenopausal women without MHT was $42.1 \pm 2.5 \mathrm{~min}$ compared with men $(\mathrm{P}=0.017)$ and postmenopausal women with MHT $(\mathrm{P}=0.002)$. With respect to SWS, postmenopausal women with MHT presented SWS significantly more than men $(5.3 \pm 0.5 \%$ versus $1.1 \pm 0.3 \%, \mathrm{P}<0.001$ ), as did postmenopausal women without MHT ( $3.9 \pm 0.4 \%, \mathrm{P}<0.001)$, although women without MHT had fewer SWS than those with MHT $(\mathrm{P}=0.049)$. NAMS (North American Menopause Society) position regarding MHT and sleep disturbances, a review of the literature in 2015 reported that low doses of estrogen or progesterone could improve chronic insomnia in postmenopausal women. At the same time, orally administered progesterone has mild sedative effects, reducing nocturnal awakenings without affecting daily cognitive functions, possibly through a GABA agonist effect.

Progesterone micronized at a dose of $300 \mathrm{mg}$ per night significantly decreases vasomotor symptoms (including daytime and nighttime hot flashes) and thus, adding its sedative effect, improves sleep quality [10]. Therefore, we conclude that MHT 
improves the subjective and objective quality of sleep, reducing the frequency of night awakenings and sleep latency time and an increasing the proportion of slow wave sleep, especially in women with vasomotor symptoms.

\section{What about Non-Hormonal Therapy?}

Any therapy that improves hot flashes could have a positive impact on the sleep quality. A Cochrane meta-analysis, which included 43 controlled and randomized clinical trials (RCTs), and 4363 participants in total, demonstrated that isoflavones would have benefit in postmenopausal women with sleep disturbances [11]. Another RCT evaluated the sleep complaint in postmenopausal women with insomnia (subjective component) and polysomnography (PSG) (objective component). One group was assigned $80 \mathrm{mg}$ daily of isoflavones and another group placebo for 4 months. Regarding the sleep complaint, it decreased from $90 \%$ to $37 \%$ in the treatment arm vs $95 \%$ to $63 \%$ in the placebo arm; on the other hand, regarding PSG parameters, an improvement in sleep efficiency of $78-84 \%$ was observed in the treatment arm vs $78-81 \%$ in the placebo arm [12].

\section{Conclusion}

Although MHT would seem to have a beneficial effect on subjective and objective sleep parameters in peri and post menopause women, its overall impact should be more extensively studied. The precise role of isoflavones and other non-hormonal treatments in sleep disturbances in this period should be established. Based on the available evidence, MHT and nonhormonal therapy are not recommended as a treatment for sleep disturbances in peri and post menopause period as an isolated symptom.

\section{References}

1. Naufel M, Frange C, Levy Andersen M, Giraro M, Tufik S, et al. (2018) Association between obesity and sleep disorders in postmenopausal women. Menopause: The Journal of The North American Menopause Society 25(2): 139-144.

2. Darien Il (2014) International Classification of Sleep Disorders, $\left(3^{\text {rd }}\right.$ edn.); American Academy of Sleep Medicine.

3. Sharples L, Glover Ma, Clutterbuck-James A, Bennet M, Jordan J, et al. (2014) Clinical effectiveness and cost-effectiveness results from the randomised controlled Trial of Oral Mandibular Advancement Devices for Obstructive sleep apnoea-hypopnoea (TOMADO) and long-term economic analysis of oral devices and continuous positive airway pressure. Health Technol Assess 18(67): 1-296.

4. Rossouw JE, Anderson GL, Prentice RL, LaCroix AZ, Kooperberg C, et al. (2002) Risk and benefits of estrogen plus progestin in healthy postmenopausal women. J Am Med Assoc 288(3): 321-333.

5. Hall M, Casement M, Troxel W, Matthews KA, Bromberger JE, set al. (2015) Chronic Stress is Prospectively Associated with Sleep in Midlife Women: The SWAN Sleep Study. Sleep 38(10):1645-1654.

6. Terauchi M, Obayashi S, Akiyoshi M, Kato K, Matsushima E, et al. (2010) Insomnia in Japanese peri- and postmenopausal women. Climacteric 13(5): 479-486.

7. JE Blumel, Cano A, Mezones-Holguin E (2012) Maturitas 72(4): 359-366.

8. Durant C, Christmas D, Nutt D (2010) The pharmacology of anxiety. Current Topics in Behavioral Neurosciences 2: 303-330.

9. Bixler E, Papaliaga M, Vgontzas A, Hung M, S Pejovic, et al. (2009) Women sleep objectively better than men and the sleep of young women is more resilient to external stressors: effects of age and menopause. J Sleep Res 18(2): 221-228.

10. Position Statement (2017) Menopause: The Journal of The North American Menopause Society 24(7): 728-753.

11. (2013) Cochrane Database Syst Rav 12.

12. Hachul H, Brandao L, D Almeida V, Bittencourt L, Baracat E, et al. (2011) Isoflavones decrease insomnia in postmenopause. Menopause 18(2): 178-184.
This work is licensed under Creative Commons Attribution 4.0 License

Submission Link: Submit Article

DOI: 10.32474/OAJRSD.2018.01.000115

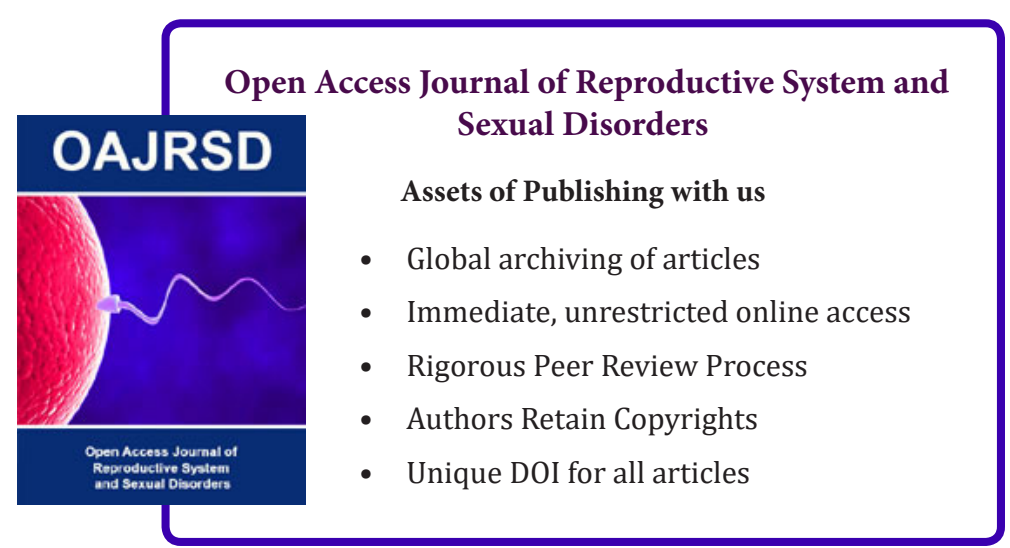

